\title{
RESIDUAL LIFETIME DISTRIBUTION AND ITS APPLICATIONS'
}

\section{M. SIDDIQUI}

Department of Statistics, Colorado State University, Colorado, U.S.A.

and

\author{
M. ÇAĞLAR
}

CEOR-Engr. Quad, Princeton University, Princeton, NJ 08544, U.S.A.

(Received for publication 29 December 1992)

Abstract- Let $T$ be a continuous positive random variable representing the lifetime of an entity. This entity could be a human being, an animal or a plant, or a component of a mechanical or electrical system. For nonliving objects the lifetime is defined as the total amount of time for which the entity carries out its function satisfactorily. The concept of aging involves the adverse effects of age such as increased probability of failure due to wear. In this paper, we consider certain characteristics of the residual lifetime distribution at age $t$, such as the mean, median, and variance, as describing aging. Gamma and Weibull families of distributions are studied from this point of view. Explicit asymptotic expressions for the mean, variance and the percentiles of corresponding residual lifetime distributions are found. Finally these families of distributions are fitted to four sets of actual data, two of which are entirely new. The results can be used in discriminating different shape parameters.

\section{Introduction}

In reliability theory, wearout failures which are a symptom of aging, occupy an important place. Several criteria for the concept of aging of a piece of equipment with a well defined survival time distribution is considered in Bryson and Siddiqui [1]. Additional criteria can also be found in Deshpande et. al. [2] In general, mean residual life (MRL) function provides a more descriptive measure of an aging process than the hazard function. They are two of the functions which completely specify the distribution [3]. Let $T$ represent the lifetime of an item and have a specified distribution function, then the distribution of $T-t$ given $T>t$ is the residual lifetime distribution

The work of the authors was carried out when the first author was a visiting professor and the second author was a graduate student at Bilkent University, Turkey during the academic year of 1990-1991. 
at time $t>0$. In this paper, residual lifetime distribution is discussed in terms of its mean, variance and percentiles as describing aging, and behavior of those quantities as $t$ tends to infinity, for certain distributions. Lawless [4] provides a relationahip between the asymptotic value of MRL and the pdf. Moreover, Calabria and Pulcini [5] provide a relationship between the asymptotic behaviors of MRL and the hazard rate. In our study of asymptotic behavior of MRL, we have additionally found the order of convergence to the limits that were found in these studies.

Because of its simplicity, Exponential distribution is widely used. This distribution has memoriless property which is equivalent to no aging in reliability context. However there are situations where the property of no memory (equivalently no aging or wearout) does not agree with the physical realities, as is the case when $T$ represents a service time or a repair time, or when failure is due to wearout. Weibull and Gamma are two typical distributions which describe the lifetime of an aging piece of equipment when the corresponding shape parameter is greater than 1 . Hence,they are investigated from the point of view mentioned, in sections 2 and 3, respectively. Finally, in section 4 , four sets of data are analyzed to apply the use of the properties of residual life distributions, such as the mean and the variance, for selecting one or more theoretical distributions which adequately fit the data.

\section{Notation}

$$
\begin{array}{ll}
T & \text { lifetime of an item } \\
f(t) & \text { pdf for lifetime } \\
R(t) & \text { reliability function, } R(t)=\operatorname{Pr}\{T>t\} \\
f_{T-t}(.) & \text { pdf for residual lifetime, } f_{T-t}(x)=f(x+t) / R(t) \\
M(.) & \text { moment generating function } \\
e(.) & \text { mean residual lifetime } \\
v(.) & \text { variance of residual lifetime } \\
\Gamma(a) & \text { Gamma function }[6,6.1] \\
\xi_{p} & p^{t h} \text { percentile of residual lifetime } \\
\beta & \text { shape parameter } \\
\lambda & \text { scale parameter } \\
Z^{+} & \text {set of positive integers }
\end{array}
$$

The pdf's of Gamma and Weibull Distributions ( $f$ and $g$ respectively) are given for 2 parameters, shape and scale, as follows:

$$
\begin{gathered}
f(x ; \beta, \lambda)=\frac{\lambda^{\beta} x^{\beta-1} e^{-\lambda x}}{\Gamma(\beta)} \beta>0, \lambda>0, x \geq 0, \\
g(x ; \beta, \lambda)=\beta \lambda(\lambda x)^{\beta-1} e^{-(\lambda x)^{\beta}} \beta>0, \lambda>0, x \geq 0 .
\end{gathered}
$$




\section{Residual Lifetime for Gamma Family of Distributions}

For the sake of simplicity, let us first consider Gamma Distribution with integer shape parameter $n$. Then, the reliability function is

$$
R(t)=\int_{t}^{\infty} \frac{\lambda^{n} x^{n-1} e^{-\lambda x}}{(n-1) !}=\sum_{k=0}^{n-1} \frac{(\lambda t)^{k} e^{-\lambda t}}{k !}
$$

and the density of the residual life time distribution is

$$
f_{T-t}(x)=\frac{\lambda^{n}(x+t)^{n-1} e^{-\lambda(x+t)}}{(n-1) ! \sum_{k=0}^{n-1} \frac{(\lambda t)^{k-2 t}}{k !}}
$$

The density function simplifies to

$$
f_{T-t}(x)=\frac{\lambda^{n}(x+t)^{n-1} e^{-\lambda x}}{(n-1) ! \sum_{k=0}^{n-1} \frac{(\lambda t)^{n}}{k !}} .
$$

The moment generating function is

$$
M(s)=\int_{0}^{\infty} \frac{e^{s x} \lambda^{n}(x+t)^{n-1} e^{-\lambda x} d x}{(n-1) ! \sum_{k=0}^{n-1} \frac{(\lambda t)^{n}}{k !}}
$$

which by integration by parts, is simplified as follows:

$$
M(s)=\frac{\left(\frac{\lambda}{\lambda-s}\right)^{n} \sum_{k=0}^{n-1} \frac{[(\lambda-\rho) t k]^{k}}{k !}}{\sum_{k=0}^{n-1} \frac{(\lambda t)^{k}}{k !}} .
$$

So the expectation of residual lifetime $e(t)$ :

$$
e(t)=\left.M^{\prime}(s)\right|_{\iota=0}=\frac{\sum_{k=0}^{n-1} \frac{(n-k) \lambda^{k-1} t^{h}}{k !}}{\sum_{k=0}^{n-1} \frac{(\lambda t)^{k}}{k !}} .
$$

The second moment:

$$
\left.M^{\prime \prime}(s)\right|_{\Theta=0}=\frac{\sum_{k=0}^{n-1} \frac{(n-k)(n-k+1) \lambda^{k-2} \ell^{k}}{k !}}{\sum_{k=0}^{n-1} \frac{(\lambda \ell)^{k}}{k !}} .
$$

As a result the variance of residual lifetime $v(t)$ :

$$
v(t)=\frac{\sum_{k=0}^{n-1} \frac{(n-k)(n-k+1) \lambda^{k-2} t^{k}}{k !}}{\sum_{k=0}^{n-1} \frac{(\lambda t)^{k}}{k !}}-\frac{\left(\sum_{k=0}^{n-1} \frac{(n-k) \lambda^{k-1} t^{k}}{k !}\right)^{2}}{\left(\sum_{k=0}^{n-1} \frac{(\lambda t)^{k}}{k !}\right)^{2}} .
$$

The percentiles of the residual life distribution at age $t$ can be found as follows. Since

$$
P\{T-t>x \mid T>t\}=\frac{\int_{x}^{\infty} \frac{\lambda^{n}(z+t)^{n-1} e^{-\lambda(s+t) d z}}{(n-1) !}}{\sum_{k=0}^{n-1} \frac{(\lambda t)^{k}}{k !} e^{-\lambda t}},
$$

$\xi_{p}$, the $p^{\text {th }}$ percentile, is the solution of the following equation in $x$ :

$$
\begin{aligned}
1-p & =P\{T-t>x \mid T>t\} \\
& =\frac{e^{-\lambda x} \sum_{k=0}^{n-1}\left(\frac{(x+)^{k} \lambda^{k}}{k !}\right.}{\sum_{k=0}^{n-1} \frac{(\lambda t)^{k}}{k !}} .
\end{aligned}
$$

This equation cannot be solved exactly for an arbitrary integer $n$. That is why the asymptotic behavior will be discussed in the next section. 


\section{Asymptotic Behavior of Mean, Variance and Percentiles of Residual Lifetime}

\section{Lifetime distribution with integer shape parameter}

For $n=1$, the residual lifetime distribution is reduced to an exponential distribution, so $e(t)=1 / \lambda \quad \forall t \geq 0$.

For $n=2$, from equation $1, e(t)=\frac{(2 / \lambda+t)}{1+\lambda t}=\frac{1}{\lambda(1+\lambda t)}+\frac{1}{\lambda}$. Then, at $t=0, e(t)=2 / \lambda$ and as $t \rightarrow \infty e(t)=1 / \lambda+O(1 / t)$.

For $n \in Z^{+}$, from equation 1 ,

$e(t)=\frac{n / \lambda+(n-1) t / 1 !+(n-2) \lambda t^{2} / 2 !+\cdots+\lambda^{n-2} t^{n-1} /(n-1) !}{1+\lambda t / 1 !+\lambda^{2} t^{2} / 2 !+\cdots+\lambda^{n-1} t^{n-1} /(n-1) !}$.

Then, at $t=0, e(t)=n / \lambda$ and as $t \rightarrow \infty \quad e(t)=1 / \lambda+O(1 / t)$.

Similarly, for the variance starting with $n=1,2$ :

For $n=1, v(t)=1 / \lambda \forall t \geq 0$.

For $n=2$, from equation 2 , at $t=0 v(t)=2 / \lambda^{2}$ and as $t \rightarrow \infty v(t)=1 / \lambda^{2}+O(1 / t)$

For $n \in Z^{+}$from equation 2 , writing the smallest and highest orders of $t$ with their coefficients in the summations:

$$
v(t)=\frac{\left(\frac{n(n+1)}{\lambda^{2}}+\cdots+\frac{(\lambda t)^{n-1}}{(n-1) !} \frac{2 \lambda^{n-2}(n-1)}{(n-1) !}\right)-\left(\frac{n^{2}}{\lambda}+\cdots+\frac{\lambda^{2(n-2) z_{2}^{2(n-1)}}}{[(n-1) !]^{2}}\right)}{1+\cdots+\frac{(\lambda t)^{2(n-1)}}{[(n-1) !]^{2}}},
$$

we obtain

$$
v(t)=\frac{\frac{n}{\lambda^{2}}+\cdots+\frac{\lambda^{2 n-4} t^{2 n-2}}{[(n-1) !]^{2}}}{1+\cdots+\frac{\lambda^{2 n-2} t^{2 n-2}}{[(n-1) !]^{2}}} .
$$

Then, at $t=0 v(t)=n / \lambda^{2}$ and as $t \rightarrow \infty \quad v(t)=1 / \lambda^{2}+O(1 / t)$, because

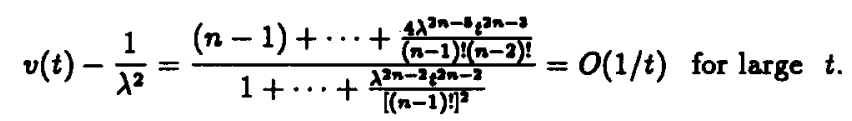

As stated in the previous section, the percentiles must also be studied for large $t$. For $n=1, \xi_{p}=-\log (1-p) / \lambda$. Let us denote the $p^{\text {th }}$ percentile corresponding to the residual lifetime with shape parameter $n$ as $\xi_{p}(n)$.

Then, for $n=2$ from equation 3 ,

$$
1-p=\frac{e^{-\lambda \xi_{0}(2)}\left[1+\left(\xi_{p}(2)+t\right) \lambda\right]}{1+\lambda t}
$$

By simplification, the following is found:

$$
\lambda \xi_{p}(2)=\log \left(1+\frac{\lambda \xi_{p}(2)}{1+\lambda t}\right)+\lambda \xi_{p}(1)
$$

But, for large $t$, the denominator of the second term in the logarithmic function also becomes large and the approximation $\log (1+x) \cong x$ can be used.

So for large $t$,

$$
\xi_{p}(2)\left[1-\frac{1}{1+\lambda t}\right] \cong \xi_{p}(1)
$$

or

$$
\xi_{p}(2)=\xi_{p}(1)+O(1 / t)
$$


For arbitrary $n$, taking logarithm of both sides of equation 3,

$\log (1-p)=-\lambda x+\log \left[1+\frac{\lambda(x+t)}{1 !}+\cdots+\frac{(\lambda(x+t))^{n-1}}{(n-1) !}\right]-\log \left[1+\frac{\lambda t}{1 !}+\cdots+\frac{(\lambda t)^{n-1}}{(n-1) !}\right]$.

By simplification and by writing the smallest and the highest orders of $t$ in the summations, we get

$$
x=\frac{1}{\lambda} \log \left[1+\frac{\lambda x+\frac{(\lambda s)^{2}}{2}+\lambda^{2} x t+\ldots+\frac{\lambda^{n-1} t^{n-2} x}{(n-2) !}}{1+\lambda t+\frac{(\lambda t)^{2}}{2}+\ldots+\frac{(\lambda t)^{n-1}}{(n-1) !}}\right]+\xi_{p}(1) .
$$

Then, using the approximation $\log (1+x) \cong x$ as we did for $n=2$ for large $t$, we have

$$
x \cong \frac{x+\lambda x^{2} / 2+\ldots+(\lambda t)^{n-2} x /(n-2) !}{1+\lambda t+\ldots+(\lambda t)^{n-1} /(n-1) !}+\xi_{p}(1) .
$$

But for large $t$, the last terms in the numerator and denominator respectively dominate, so that

$$
x \cong \frac{(\lambda t)^{n-2} x /(n-2) !}{(\lambda t)^{n-1} /(n-1) !}+\xi_{p}(1)
$$

This gives

$$
x\left[1-\frac{n-1}{\lambda t}\right] \cong \xi_{p}(1)
$$

or finally,

$$
x=\xi_{p}(1)\left[1+\frac{n-1}{\lambda t}+O\left(1 / t^{2}\right)\right]
$$

The last equation is written by the identity $\frac{1}{1-=}=\sum_{k=0}^{\infty} x^{k}$ for sufficiently small $x>0$. Now $x$ stands for $\xi_{p}(n)$, so for large $t, \xi_{p}(n)=\xi_{p}(1)+O(1 / t)$.

These are interesting results, because this means that if an item whose lifetime distribution is defined by a Gamma Distribution (with integer shape parameter) survives a long time, then it behaves agymptotically as if it has an exponential residual lifetime distribution, i.e., it behaves as if it does not age any more. Moreover, for $n \in\{1,2, \ldots\}$ it can be concluded that both $e(t)$ and $v(t)$ are decreasing functions of $t$. And for large $t$, the order of decrease is $O(1 / t)$.

\section{Generalization to real shape parameter}

The mean residual lifetime in general is

$$
e(t)=\frac{\int_{t}^{\infty} x f(x) d x}{R(t)}-t
$$

By writing the denaity and the reliability functions explicitly in 4 and by simplification, we get

$$
e(t)=\frac{\int_{t}^{\infty} x^{\rho} e^{-\lambda x} d x}{\int_{t}^{\infty} x^{\beta-1} e^{-\lambda x}}-t .
$$

After this point, we apply integration by parts to the integral in the numer- 
ator, and calling the integral in the denominator $I_{1}$, the result in terms of $I_{1}$ only, is:

$$
e(t)=\frac{1}{\lambda}+\frac{(\beta-1) I_{1}+t^{\beta} e^{-\lambda t}-\lambda t I_{1}}{\lambda I_{1}} .
$$

Now, let $I_{k}=\int_{t}^{\infty} x^{\rho-k} e^{-\lambda \varepsilon} d x$ and apply integration by parts several times:

$$
e(t)=\frac{1}{\lambda}+\frac{\frac{(\beta-1) \rho-2}{\lambda^{2}}+\frac{(\beta-1)^{2}(\rho-2) e^{\lambda t}}{\lambda^{2}} I_{3}-\frac{(\beta-1)(\rho-2)(\rho-3) t e^{\lambda t}}{\lambda^{2}} I_{4}}{t^{\beta-1}+(\beta-1) e^{\lambda t} I_{2}} .
$$

Finally, for large $t$,

$$
e(t)=\frac{1}{\lambda}+O\left(\frac{\beta-1}{\lambda^{2} t}\right)=\frac{1}{\lambda}+O\left(\frac{1}{t}\right)
$$

as in the case of integer shape parameter.

The variance for the general case is studied in the same manner. It is explicitly:

$$
\begin{aligned}
v(t) & =E\left\{T^{2} \mid T>t\right\}-E^{2}\{T \mid T>t\} \\
& =\frac{\int_{t}^{\infty} x^{2} f(x) d x}{R(t)}-\frac{\left(\int_{t}^{\infty} x f(x) d x\right)^{2}}{R^{2}(t)} .
\end{aligned}
$$

Putting the expressions of $R(t)$ and $f(x)$,and applying integration by parts several times, we get

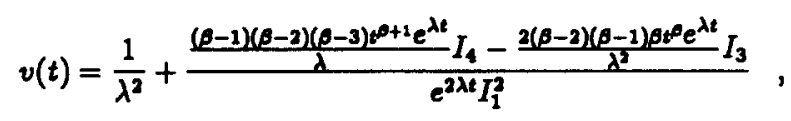

so that

$$
v(t)=\frac{1}{\lambda^{2}}+\frac{O\left(t^{2 \beta-3}\right)}{O\left(t^{2 \beta-2}\right)}=\frac{1}{\lambda^{2}}+O\left(\frac{1}{t}\right) .
$$

Percentiles of the residual lifetime distribution can be obtained in the same way as the percentiles for integer shape parameter. Let $n \leq \beta<n+1$.

Then the real shape parameter version of 3 is

$$
1-p=\left(\int_{z}^{\infty} \frac{\lambda^{\beta}(z+t)^{\beta-1} e^{-\lambda(z+t)}}{\Gamma(\beta)} d z\right) /\left(\int_{t}^{\infty} \frac{\lambda^{\beta} z^{\beta-1} e^{-\lambda z}}{\Gamma(\beta)} d z\right) .
$$

Putting $u=z+t$, and simplifying the righthand side, we obtain

$$
1-p=\frac{\int_{x+t}^{\infty} u^{\beta-1} e^{-\lambda u} d u}{\int_{t}^{\infty} z^{\beta-1} e^{-\lambda z} d z}
$$

By integration by parts several times, it is found that

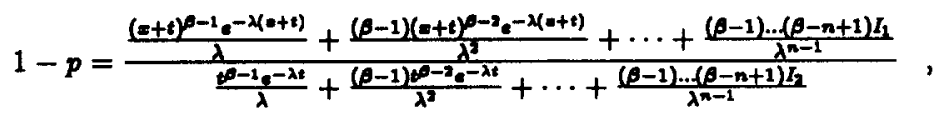

where

$$
\begin{aligned}
& I_{1}=\int_{x+t}^{\infty} u^{\rho-n} e^{-\lambda u} d u \\
& I_{2}=\int_{t}^{\infty} u^{\rho-n} e^{-\lambda u} d u
\end{aligned}
$$

Let's call the numerator of last equation as $a$ and the denominator as $b$.

Then taking the logarithm of both sides,

$$
\log (1-p)=-\lambda(x+t)+\log \left(e^{\lambda(x+t)} a\right)+\lambda t-\log \left(e^{\lambda t} b\right) .
$$


By rearranging the logarithm terms on the righthand side and writing the smallest and highest orders of $t$ in the summations,

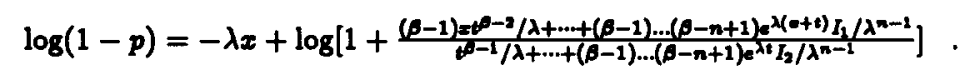

Because, for large $t$, the algebraic term inside the logarithm function in the last equation becomes small; we can use the approximation $\log (1+x) \cong x$.

As a result,

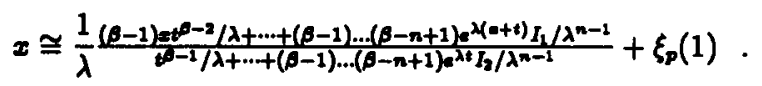

Moreover for large $t$, the highest orders of $t$ are the dominating terms in both the numerator and the denominator, so that

$$
x \cong \frac{1}{\lambda} \frac{(\beta-1) x t^{\beta-2} / \lambda}{t^{\beta-1} / \lambda}+\xi_{p}(1)
$$

or

$$
x \cong \frac{(\beta-1) x}{\lambda t}+\xi_{p}(1)
$$

and finally

$$
x=\left[1+\frac{\beta-1}{\lambda t}+O\left(1 / t^{2}\right)\right] \xi_{p}(1) \forall \beta>0
$$

So, all results pertaining to integer shape parameter case are also valid for real shape parameter. Moreover, these results suggest that, in model selection we have to study the MRL plots for relatively small $t$, in order to discriminate between different shape parameters of Gamma distribution, because for large $t$ they all converge to $1 / \lambda$ and thus discrimination becomes difficult. What is more, the results can be used in debugging or burn-in processes. For example, some trade off can be found in terms of the testing period; the MRL of the items decreases when they are debugged, but on the contrary the residual lifetime becomes more stable in terms of both its mean and the variance.

\section{Residual Lifetime for Weibull Family of Distributions}

The residual lifetime density is

$$
f_{T-t}(x)=\frac{\beta \lambda^{\beta}(x+t)^{\beta-1} e^{-[\lambda(x+t)]^{\rho}}}{e^{-(\lambda t)^{\rho}}} .
$$

The moment generating function is

$$
M(s)=\frac{\int_{0}^{\infty} e^{\alpha s}[\lambda(x+t)]^{\beta-1} e^{-[\lambda(x+t)]^{\beta} \lambda \beta d x}}{e^{-(\lambda t)^{\rho}}},
$$

by uring the identity $e^{x}=\sum_{i=0}^{\infty} \frac{\varepsilon^{i}}{i !}$ and by making a change of variable $u=$ $[\lambda(x+t)]^{e}$, we get

$$
M(s)=\frac{e^{-s t}}{e^{-(\lambda t)}} \sum_{i=0}^{\infty}(s / \lambda)^{i} \frac{\int_{(\lambda t) u^{i / \beta} e^{-u} d u}^{\infty} \Gamma(i+1)}{\Gamma(i+1)} .
$$


Let

$$
\Gamma(a, x)=\int_{x}^{\infty} u^{a-1} e^{-u} d u
$$

Then the moment generating function is found as

$$
M(s)=\frac{e^{-a t}}{e^{-(\lambda t)}} \sum_{i=0}^{\infty}(s / \lambda)^{i} \frac{\Gamma\left(i / \beta+1,(\lambda t)^{\beta}\right)}{\Gamma(i+1)}
$$

and mean residual lifetime is

$$
e(t)=\left.M^{\prime}(s)\right|_{\rho=0}=\frac{\Gamma\left(1+1 / \beta,(\lambda t)^{\beta}\right)}{\lambda e^{-(\lambda t)^{\rho}}}-t .
$$

The second moment is

$$
\left.M^{\prime \prime}(s)\right|_{\ominus=0}=t^{2}-\frac{2 t \Gamma\left(1+1 / \beta,(\lambda t)^{\beta}\right)}{\lambda e^{-(\lambda t)^{\rho}}}+\frac{\Gamma\left(1+2 / \beta,(\lambda t)^{\beta}\right)}{\lambda^{2} e^{-(\lambda t)^{\beta}}},
$$

so the variance is found as

$$
v(t)=\frac{\Gamma\left(1+2 / \beta,(\lambda t)^{\beta}\right)}{\lambda^{2} e^{-(\lambda t)^{\beta}}}-\frac{\Gamma^{2}\left(1+1 / \beta,(\lambda t)^{\beta}\right)}{\lambda^{2} e^{-2(\lambda t)^{\beta}}} .
$$

The $p^{\text {th }}$ percentile $\xi_{p}$ is the solution of the following equation in $x$ :

$$
p=P\{T-t \leq x \mid T>t\}=\frac{\int_{0}^{x} \beta \lambda[\lambda(y+t)]^{\beta-1} e^{-[\lambda(y+t))^{\rho}} d y}{e^{-(\lambda t)^{\mathcal{\rho}}}} .
$$

Evaluating the integral in the last expression by putting $u=[\lambda(y+t)]^{\beta}$, we get

$$
p=\frac{e^{-(\lambda t)^{\rho}}-e^{-[\lambda(x+t)]^{p}}}{e^{(-\lambda t)^{\rho}}},
$$

and finally

$$
\xi_{p}=t\left[1-\frac{\log (1-p)}{(\lambda t)^{\beta}}\right]^{1 / \beta}-t
$$

\section{Asymptotic Behavior of Expectation, Variance and Per- centiles of Residual Lifetime Distribution}

$$
\begin{gathered}
\text { At } t=0, e(t)=\frac{\Gamma(1+1 / \beta)}{\lambda}, \text { which is the expected value of } T \text {. For } t>0, \\
e(t)=\frac{\Gamma\left(1+1 / \beta,(\lambda t)^{\beta}\right)}{\lambda e^{-(\lambda t)^{\beta}}}-t
\end{gathered}
$$

and in open form, $\Gamma\left(1+1 / \beta,(\lambda t)^{\beta}\right)=\int_{(\lambda t)^{\rho}}^{\infty} u^{1 / \beta} e^{-u} d u$. By integration by parts, this can be reduced to

$$
\Gamma\left(1+1 / \beta,(\lambda t)^{\beta}\right)=\lambda t e^{-(\lambda t)^{\beta}}+\int_{\lambda t}^{\infty} e^{-x^{\beta}} d x .
$$

Let $I_{1}=\int_{\lambda t}^{\infty} \mathrm{e}^{-x^{\circ}} \mathrm{dx} . I_{1}$ cannot be evaluated but an approximation for large $t$ can be found. By integration by parts, it can be shown that

$$
\frac{e^{-(\lambda t)^{\rho}}}{\beta(\lambda t)^{\beta-1}}\left[1-\frac{\beta-1}{\beta(\lambda t)^{\beta}}\right] \leq I_{1} \leq \frac{e^{-(\lambda t)^{\beta}}}{\beta(\lambda t)^{\beta-1}}
$$

Then, for large $t$,

$$
I_{1}=\frac{e^{-(\lambda t)^{\rho}}}{\beta(\lambda t)^{\beta-1}}\left[1+O\left(1 / t^{\beta}\right)\right]
$$


By putting this first in equation 8 , then in equation 7 , and by simplification, for large $t$, we obtain:

$$
e(t)=\frac{1}{\beta \lambda(\lambda t)^{\beta-1}}\left[1+O\left(1 / t^{\beta}\right)\right]
$$

This last expression is valid for large $t$ only, but it is much more practical for computation than equation 7 .

So

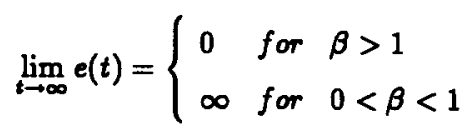

And for $\beta=1, e(t)=1 / \lambda$.

The variance (in equation 5 ) can be studied in a similar fashion, then in open form,

$$
\Gamma\left(1+2 / \beta,(\lambda t)^{\beta}\right)=\int_{(\lambda t) \rho}^{\infty} u^{2 / \beta} e^{-u} d u .
$$

By a change of variable $u=x^{\beta}$ and by integration by parts, we have

$$
\Gamma\left(1+2 / \beta,(\lambda t)^{\beta}\right)=\lambda^{2} t^{2} e^{-(\lambda t)^{\beta}}+2 \int_{\lambda t}^{\infty} x e^{-x^{\beta}} d x .
$$

Let $I_{2}=\int_{\lambda t}^{\infty} x e^{-x^{\infty}} d x$. For large $t, I_{2}$ can be approximated as

$$
I_{2}=\frac{e^{-(\lambda t)^{\rho}}}{\beta(\lambda t)^{\beta-2}}\left[1+O\left(1 / t^{\beta}\right)\right]
$$

Cancellation occurs with $\Gamma^{2}\left(1+1 / \beta,(\lambda t)^{\beta}\right)$ in which there is $I_{1}$, which is also approximated as in equation 9 . So, one more step is taken in approximation for both $I_{1}$ and for $I_{2}$. The results are as follows:

$$
\begin{aligned}
& I_{1}=\frac{e^{-(\lambda t)^{\beta}}\left(\beta(\lambda t)^{\beta}-\beta+1\right)}{\beta^{2}(\lambda t)^{2 \beta-1}}\left[1+O\left(1 / t^{2 \beta}\right)\right] ; \\
& I_{2}=\frac{e^{-(\lambda t)^{\beta}}\left(\beta(\lambda t)^{\beta}-\beta+2\right)}{\beta^{2}(\lambda t)^{2 \beta-2}}\left[1+O\left(1 / t^{2 \beta}\right)\right] .
\end{aligned}
$$

Putting those expressions in equation 8 and equation 10 respectively, then putting the results in equation 5 , we obtain

$$
v(t)=\frac{\beta(\lambda t)^{\beta}\left[\beta(\lambda t)^{\beta}+2(\beta-1)\right]-(1-\beta)^{2}}{\lambda^{2} \beta^{4}(\lambda t)^{4 \beta-2}}\left[1+O\left(1 / t^{2 \beta}\right)\right]
$$

and

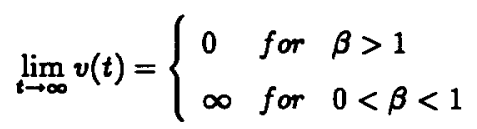

For $\beta=1, v(t)=1 / \lambda^{2}$.

The asymptotic behavior of the percentiles can be studied in relation to equation 6 as follows, using the fact that $-\log (1-p)<(\lambda t)^{2}$ for large $t$, by the binomial expansion formula,

$\xi_{p}=t\left[1-\frac{1}{\beta} \frac{\log (1-p)}{1 !(\lambda t)^{\beta}}+\frac{1}{\beta}\left(\frac{1}{\beta}-1\right) \frac{\log ^{2}(1-p)}{2 !(\lambda t)^{2 \beta}}-\frac{1}{\beta}\left(\frac{1}{\beta}-1\right)\left(\frac{1}{\beta}-2\right) \frac{\log ^{3}(1-p)}{3 !(\lambda t)^{3 \beta}}+\cdots\right]-t$,

so that

$\xi_{p}=-\frac{t}{\beta} \frac{\log (1-p)}{(\lambda t)^{\beta}}\left[1-\left(\frac{1}{\beta}-1\right) \frac{\log (1-p)}{2 !(\lambda t)^{\beta}}+\left(\frac{1}{\beta}-1\right)\left(\frac{1}{\beta}-2\right) \frac{\log ^{2}(1-p)}{3 !(\lambda t)^{2 \beta}}-\cdots\right]$ 


$$
=-\frac{t \log (1-p)}{\beta(\lambda t)^{\beta}}\left[1+O\left(1 / t^{\beta}\right)\right] .
$$

So

$$
\lim _{t \rightarrow \infty} \xi_{p}=\left\{\begin{array}{ll}
0 & \text { for } \beta>1 \\
\infty & \text { for } 0<\beta<1
\end{array} .\right.
$$

The above result is valid for $\beta \neq 1$. For $\beta=1$, the distribution is reduced to the exponential distribution which has been discussed in the previous section.

Having found the expressions for large $t$, for $e(t), v(t)$, and $\xi_{p}$ we can conclude that the greater the shape parameter the faster the convergence to 0 (when $\beta>1$ ), and the same is valid for the variance and the percentiles of the residual lifetime. In other words, when the item's lifetime is explained by Weibull distribution, the aging process is much severe for larger shape parameters. Hence, this property can be considered in discrimination of different shape parameters of Weibull distribution or in discrimination between different types of distributions.

\section{Data Analysis and Conclusion}

In this chapter, four different sets of data are studied for the purpose of applying the mean residual lifetime function as a criterion for aging. The first two sets are lifetimes of 75 Watt bulbs produced in two different Turkish factories; the third one is the lifetime of Kevlar 49/Epoxy Strands [7] (tested at $70 \%$ stress level), and the last one is the service time between failures of the air-conditioning equipment in Boeing 720 jet aircraft [8]. For this last set, the essential assumption is that after repair the equipment becomes as good as new. The first three sets of data are examples of items with decreasing mean residual life, i.e., the items are aging in time. Conversely, the last data set is an example of exponentially distributed time between failures (lifetimes, in a sense). The reason for selecting decreasing mean residual lifetime distributions is the greater importance of aging items in production environment than those with no aging, in terms of quality. The data are given in tables 1 and 2 .

At this point it must be stated that data set 1 and data set 2 are ordered statistics taken from random samples, tested under 320 Volts and 286 Volts, respectively, voltages which are never encountered during normal usages of those bulbs. These high voltages are only for shortening the test period and observing all of the bulbs' lifetime, since under 220 Volts the lifetime of a bulb may be in years. 
The natural estimator for the mean residual lifetime function is:

$$
\hat{e}(t)=S^{-1} \sum_{j=1}^{s}\left(t_{j}-t\right),
$$

where $S$ denotes the number of survivors at time t out of an initial population of size $N$, and $\left\{t_{j}: j=1, \ldots, S\right\}$ is the set of data points which are greater than $t$.

Since the first three data sets show decreasing mean residual lifetime, Gamma and Weibull distributions were fitted to them. Moreover they reveal the presence of a truncation parameter, because the smallest value in the set is comparatively high. We fitted three parameter Weibull and Gamma distributions. In estimation procedure, we estimated truncation parameter by the smallest data point (let it be $x_{1}$ ), then transferred the data to a new one by subtracting $x_{1}$ from each data point. Then we fitted two parameter Weibull and Gamma distributions to the transferred data set. We have used moment estimators.

Table 1: The Data Sets (description is given in the text)

\begin{tabular}{|c|c|c|c|}
\hline Deta Set 1 (min.) & Data Set 2 (hr.) & Data Set 3 (hr.) & Data Set 4 (hr.) \\
\hline 295 & 750 & 1051 & 3 \\
\hline 420 & 778 & 1337 & 5 \\
\hline 420 & 865 & 1389 & 5 \\
\hline 445 & 904 & 1921 & 13 \\
\hline 450 & 956 & 1942 & 14 \\
\hline 470 & 983 & 2322 & 15 \\
\hline 470 & 988 & 3629 & 22 \\
\hline 500 & 1000 & 4006 & 22 \\
\hline 500 & 1034 & 4012 & 23 \\
\hline 502 & 1061 & 4063 & 30 \\
\hline 525 & 1063 & 4921 & 36 \\
\hline 540 & 1063 & 5445 & 39 \\
\hline 550 & 1065 & 5620 & 44 \\
\hline $\mathbf{5 5 0}$ & 1097 & 5817 & 46 \\
\hline 555 & 1100 & 5905 & 50 \\
\hline 555 & 1108 & 5956 & 72 \\
\hline 560 & 1116 & 6068 & 79 \\
\hline 570 & 1124 & 6121 & 88 \\
\hline 580 & 1179 & 6473 & 97 \\
\hline 580 & 1210 & 7501 & 102 \\
\hline 600 & 1214 & 7886 & 139 \\
\hline 605 & 1222 & 8108 & 188 \\
\hline 610 & 1285 & 8546 & 197 \\
\hline 630 & 1297 & 8666 & 210 \\
\hline 630 & 1308 & 8831 & \\
\hline 645 & 1308 & 9106 & \\
\hline 660 & 1380 & 9711 & \\
\hline 660 & 1399 & 9806 & \\
\hline 675 & 1415 & 10205 & \\
\hline 685 & 1466 & 10396 & \\
\hline 690 & 1484 & 10861 & \\
\hline 690 & 1533 & 11026 & \\
\hline 690 & 1533 & 11214 & \\
\hline 695 & 1580 & 11362 & \\
\hline 700 & 1612 & 11604 & \\
\hline $\begin{array}{l}715 \\
715\end{array}$ & $\begin{array}{l}1698 \\
1698\end{array}$ & $\begin{array}{l}11608 \\
11745\end{array}$ & \\
\hline 720 & 1765 & 11762 & \\
\hline 720 & 1824 & 11895 & \\
\hline 725 & 1946 & 12044 & \\
\hline
\end{tabular}

Table 2: The Data Sete Continued

\begin{tabular}{|c|c|c|}
\hline Data Set 1 & Data Set 2 & Date Set 3 \\
\hline 750 & 1946 & 13520 \\
\hline 755 & 1968 & 13670 \\
\hline 768 & 2005 & 14110 \\
\hline 780 & 2005 & 14496 \\
\hline 780 & 2005 & 15395 \\
\hline 785 & 2264 & 16178 \\
\hline 800 & 2314 & 17092 \\
\hline 810 & 2319 & 17568 \\
\hline 810 & 2332 & 17568 \\
\hline 830 & 2458 & \\
\hline 840 & & \\
\hline 870 & & \\
\hline 870 & & \\
\hline 870 & & \\
\hline $\begin{array}{l}885 \\
885\end{array}$ & & \\
\hline $\begin{array}{l}885 \\
890\end{array}$ & & \\
\hline 900 & & \\
\hline 909 & & \\
\hline 910 & & \\
\hline 915 & & \\
\hline 915 & & \\
\hline 915 & & \\
\hline 930 & & \\
\hline 950 & & \\
\hline 960 & & \\
\hline $\begin{array}{l}960 \\
960\end{array}$ & & \\
\hline 960 & & \\
\hline 890 & & \\
\hline 990 & & \\
\hline 1005 & & \\
\hline 1005 & & \\
\hline 1020 & & \\
\hline 1050 & & \\
\hline $\begin{array}{l}1050 \\
1080\end{array}$ & & \\
\hline 1185 & & \\
\hline 1200 & & \\
\hline 1200 & & \\
\hline
\end{tabular}


For random variable $X$ distributed with Weibull distribution, let $\gamma$ be the truncation parameter. Then the density function is:

$$
g(x ; \beta, \lambda, \gamma)=\beta \lambda^{\beta}(x-\gamma)^{\beta-1} e^{-[\lambda(x-\gamma)]^{\beta}} \quad \gamma<x<\infty, \beta>0, \lambda>0
$$

The interpretation of $\gamma$ can be some type of guarantee period, in our concern. Then we estimate it as $\hat{\gamma}=x_{1}$. The first two moments of the distribution are:

$$
E\left\{X-x_{1}\right\}=\frac{\Gamma_{1}}{\lambda} \quad V\left\{X-x_{1}\right\}=\frac{\Gamma_{2}-\Gamma_{1}^{2}}{\lambda^{2}}
$$

where $\Gamma_{k}=\Gamma(1+k / \beta)$.

On equating the first two sample moments to corresponding distribution moments as given above, the moment estimators become

$$
\frac{\hat{\Gamma_{1}}}{\hat{\lambda}}=\bar{x}-x_{1}, \quad \frac{\hat{\Gamma_{2}}-\hat{\Gamma}_{1}^{2}}{\hat{\lambda}^{2}}=s^{2} \text {. }
$$

In $[9$, page 30$]$, the $\beta$ values versus the coefficient of variation (C.V.) are tabulated. So the estimation procedure is simplified by first finding an approximation for $\hat{\beta}$ from this table, by considering the sample coefficient of variation. This approximation is used to determine $\hat{\lambda}$ from either of the equations in 11. It is then, improved by trial and error until both of them gave the same result for $\hat{\lambda}$.

For Gamma distribution, with truncation parameter $\gamma$,

$$
f(x ; \beta, \lambda, \gamma)=\frac{\lambda^{\beta}(x-\gamma)^{\beta-1} e^{-\lambda(x-\gamma)}}{\Gamma(\beta)} \quad \gamma<x<\infty, \beta>0, \lambda>0 .
$$

As explained before, $\hat{\gamma}=x_{1}$. The first two moments are:

$$
E\left\{X-x_{1}\right\}=\frac{\beta}{\lambda} \quad V\left\{X-x_{1}\right\}=\frac{\beta}{\lambda^{2}} .
$$

On equating the first two sample moments to corresponding distribution moments, the moment estimators become

\begin{tabular}{|c|c|c|c|c|}
\hline \multicolumn{5}{|c|}{ Data Sets } \\
\hline 1 & \multicolumn{2}{|c|}{2} & \multicolumn{2}{|c|}{3} \\
\hline Gamma & Weibull & Gamma & Weibull & Gamma \\
\hline 5.22 & 1.55 & 2.29 & 1.75 & 2.9 \\
\hline 0.0113 & 0.0013 & 0.0033 & 0.000115 & 0.000374 \\
\hline 295 & 750 & 750 & 1051 & 1051 \\
\hline
\end{tabular}

$$
\hat{\lambda}=\frac{\bar{x}-x_{1}}{s^{2}} \text { and } \hat{\beta}=\hat{\lambda}\left(\bar{x}-x_{1}\right)=\frac{\left(\bar{x}-x_{1}\right)^{2}}{s^{2}}
$$

The results are given in Table 3 . As it can be observed from this table, the shape parameters are strictly greater than 1 , so the items are aging in time.

Table 3: Estimated values of parameters 
The reliability and MRL functions are plotted in figures 1 to 2, 3 to 4, and 5 to 6 for the data sets 1,2 and 3, respectively. The empirical reliability function is found from the following equation:

$$
\hat{R}(t)=\frac{S}{N}
$$

where $S$ and $N$ are as defined before.

Moreover, in the graphs of $e(t)$ versus $t$, the upper bound (UB) and lower bound (LB) are found to be the approximate $95 \%$ s-confidence limits as follows:

$$
\begin{aligned}
& U B=\hat{e}(t)+2 \frac{s(t)}{\sqrt{S}} \\
& L B=\hat{e}(t)-2 \frac{s(t)}{\sqrt{S}},
\end{aligned}
$$

where $s(t)$ is the estimated standard deviation of the residual lifetime at $t$, i.e.

$$
s(t)=(S-1)^{-1} \sum_{j=1}^{S}\left[t_{j}-e(t)\right]^{2} .
$$

The last data set was fitted a Gamma Distribution using MLE's in [8] without assuming a truncation parameter, and $\hat{\beta}$ and $\hat{\lambda}$ were found to be 1.06 and 0.0156 , respectively. So we can consider this set as a sample from an exponential distribution with parameter 0.0156 . The reliability and MRL functions are plotted in figures 7 and 8 . The reliability plot fits well and this is supported by MRL plot which remains quite constant in time.

In order to test the goodness of fit, Kolmogorov-Smirnov statistic $D_{N}$ is used. It is defined as [10]

$$
D_{N}=\sup _{t}\left|R_{N}(t)-R(t)\right|,
$$

where $N$ is the sample size. The distribution of $D_{N}$ is independent of the cdf $F(t) \quad(=1-R(t))$ that defines $H_{0}$. Accordingly, the acceptance limits for the test of Goodness of Fit are tabulated in [10, page 580]. The critical region $D_{N}>D_{N, a}$ is used to test $F(t)$ against the alternative that the cdf is not $F(t)$, where $D_{N, \alpha}$ is the constant corresponding to the 8-significance level $\alpha$. The values of test statistic are given in Table 4 for all the data sets, for $\alpha=.05$. With those values both Weibull and Gamma distributions are accepted for the first three sets, and the one whose $D_{N}$ value is smaller, is regarded as the better fitting distribution and marked with a star. With this criterion, in all of them Weibull distribution came out to be better. But we can comment that for the second data set, considering the MRL plot (Figure 4), Gamma distribution might be preferred, because $\hat{e}(t)$ does not decrease, but rather fluctuates after some time, and this obeys to our results for Gamma distribution in Section 2. In fact, for this set the KolmogorovSmirnov test atatistics for the two distributions are very close to eachother. Finally, for the last set Exponential distribution is also accepted. 
We can then, conclude that for the first three data sets, items are aging in time, and exponential distribution cannot explain the behavior of the bulbs' lifetimes. From the graphs of $e(t)$ versus $t$, it can be seen that the theoretical curves fit the emprical results, quite well. In relevant situations such as this, MRL function being one of the distribution identities, can also be used as a diagnostic procedure to make a distinction among different distributions, together with the reliability function.

Table 4: Kolmogorov-Smirnov Test Statistic Values

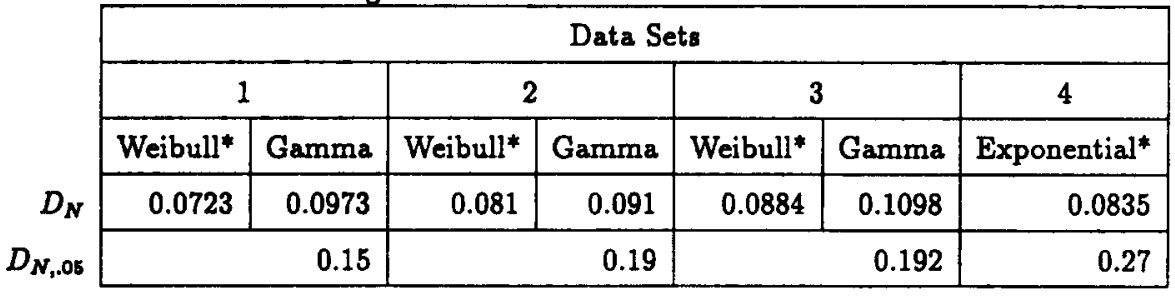

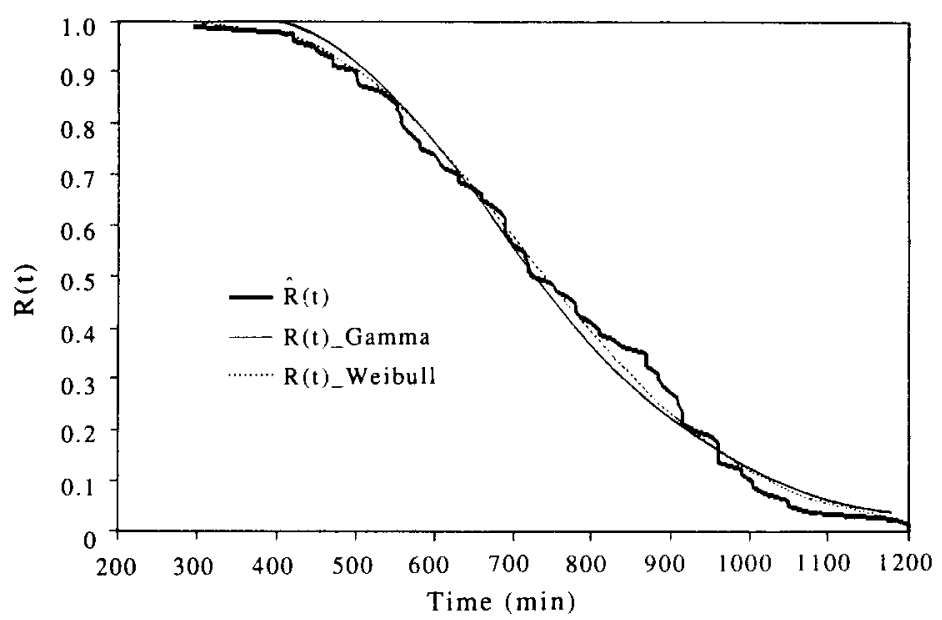

1. The fitted and empirical $(\hat{R}(t))$ reliability functions for Data Set 1

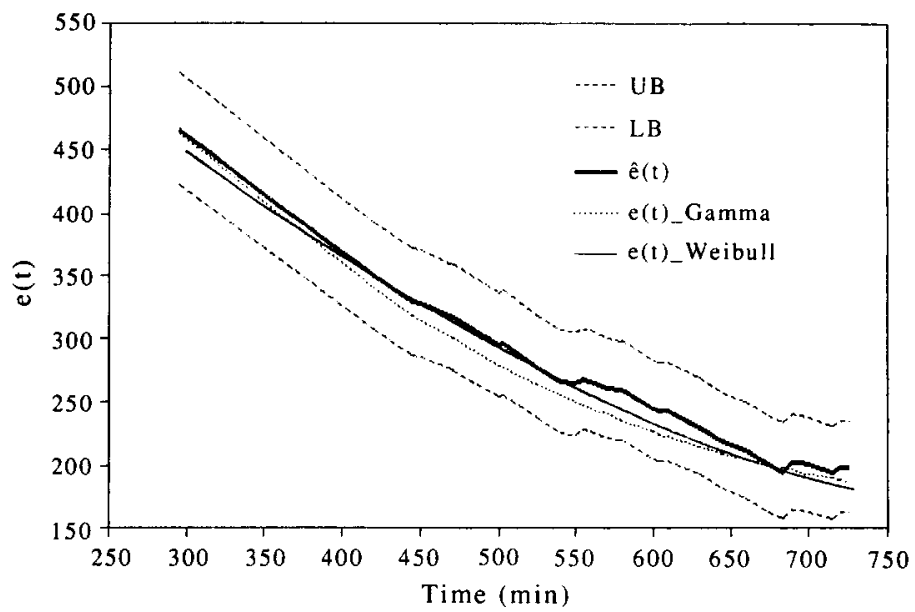

2. The fitted Gamma and Weibull $e(t)$ and the empirical $\hat{e}(t)$ for Data Set 1 


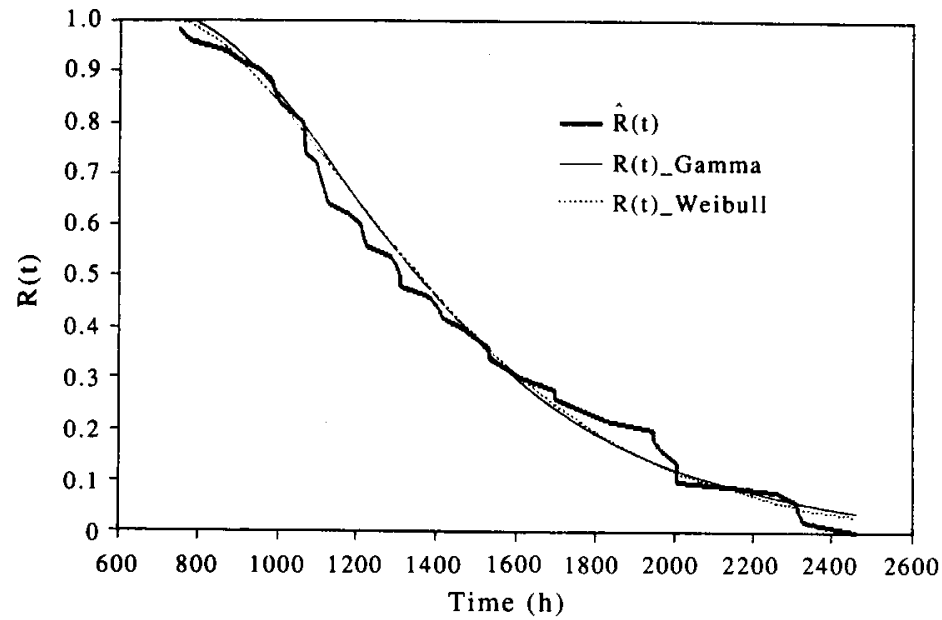

3. The fitted and empirical $(\hat{R}(t))$ reliability functions for Data Set 2

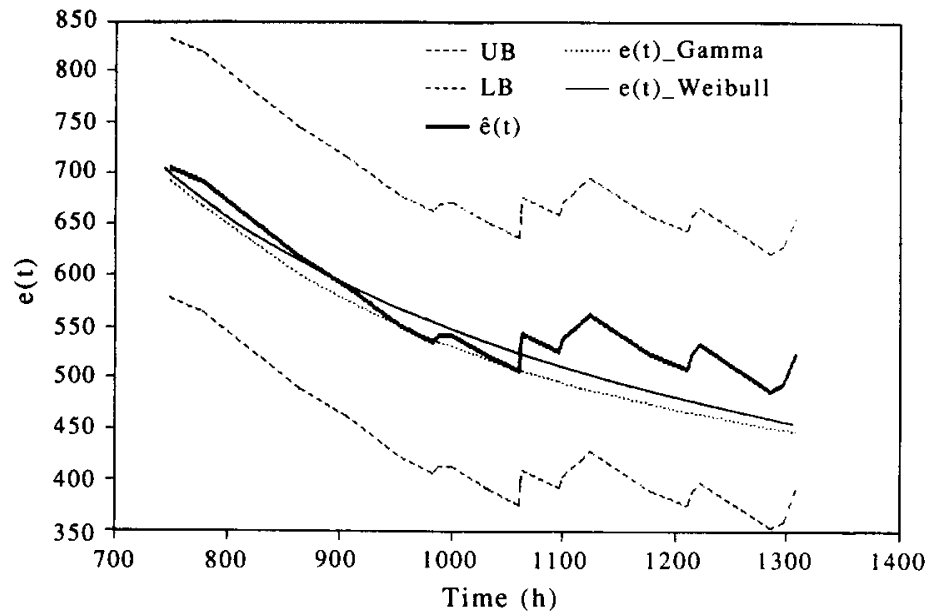

4. The fitted Gamma and Weibull $e(t)$ and the empirical $\hat{e}(t)$ for Data Set 2

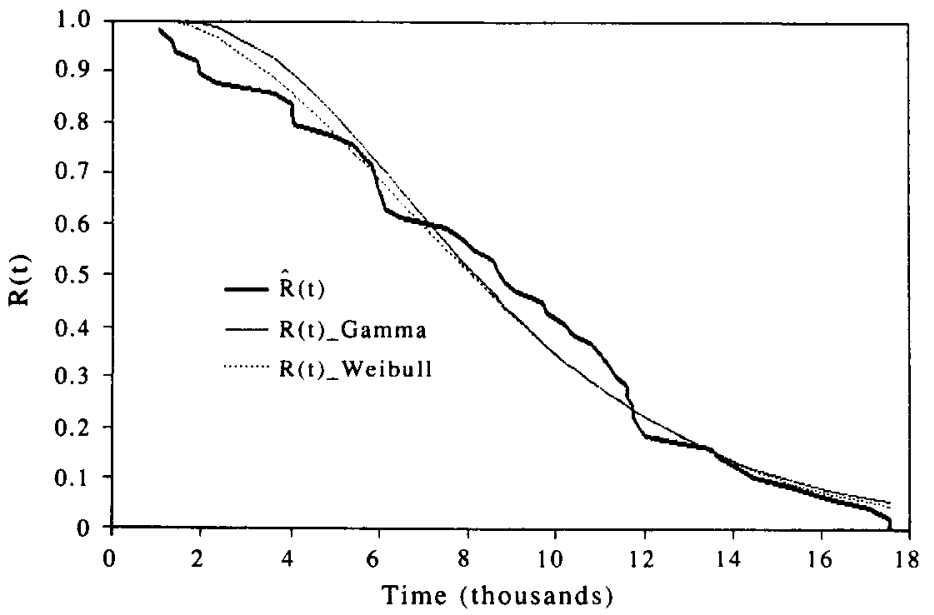

5. The fitted and empirical $(\hat{R}(t))$ reliability functions for Data Set 3 


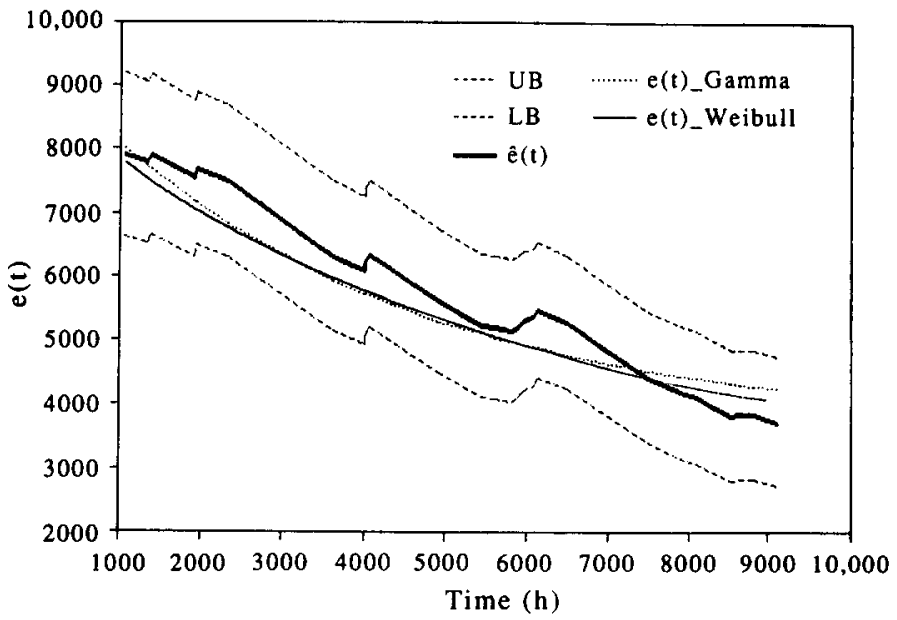

6. The fitted Gamma and Weibull $e(t)$ and the empirical $\hat{e}(t)$ for Data Set 3

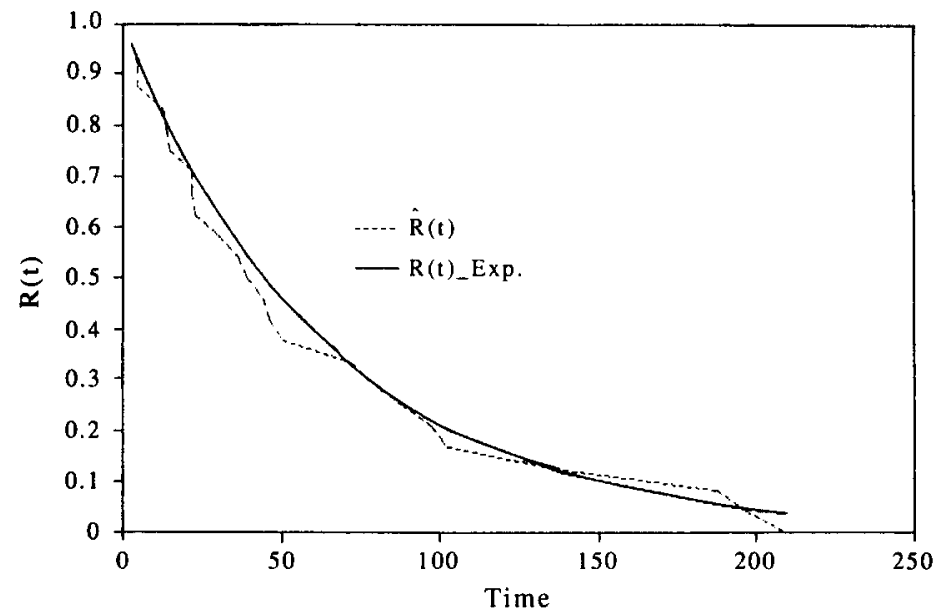

7. The fitted and empirical $(\hat{R}(t))$ reliability functions for Data Set 4

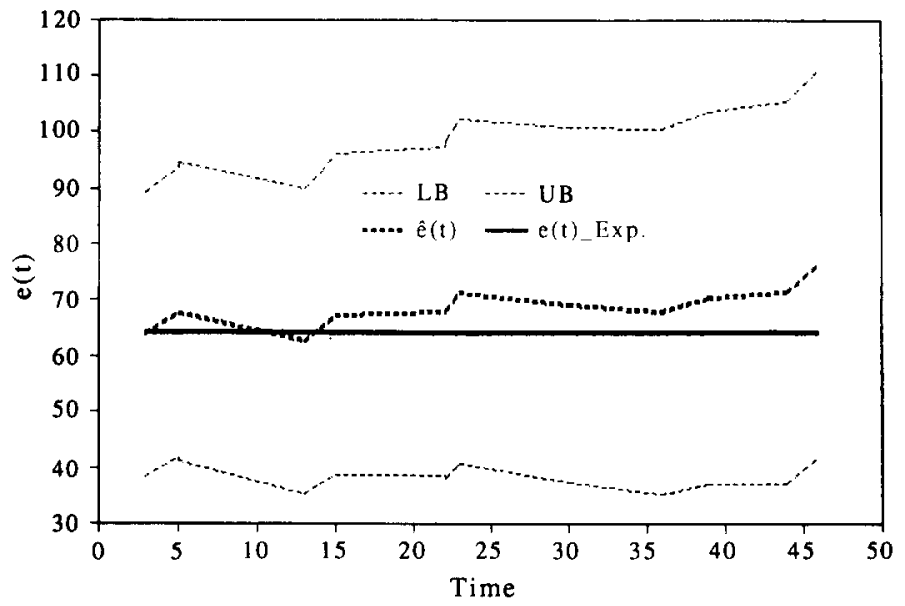

8. The fitted Exponential $e(t)$ and the empirical $\hat{e}(t)$ for Data Set 4 


\section{References}

[1] M. C. Bryson, M. M. Siddiqui, J. Amer. Statist. Assn. 64, 1472-1483 (1969).

[2] J. V. Deshpande, et. al., J. Appl. Probab. 23, 748-758 (1986).

[3] L. M. Leemis, IEEE Trans. Rel. R-35, 170-174 (1980).

[4] J. F. Lawless, Statistical Models and Methods for Lifetime Data, John Wiley and Sons, New York (1982).

[5] R. Calabria,G. Pulcini, Reliab. Eng. 19, 165-170 (1987).

[6] M. Abramowitz; I. A. Stegun, Handbook of Mathematical Functions, Dover Publications, Inc., New York (1982).

[7] D. F. Andrews, A. M. Herzberg, DATA A Collection of Problems from Many Fields for the Student and the Research Worker, Springer-Verlag, New York (1985).

[8] D. R. Cox, E. J. Snell, Applied Statistics, Cambridge University Press, London (1989).

[9] A. C. Cohen, B. J. Whitten, Parameter Estimation in Reliability and Life Span Models, Marcell Dekker Inc., New York (1988).

[10] B. W. Lindgren, Statistical Theory, Macmillan Publishing Co. Inc., New York (1976). 